\title{
The biological and geological contingencies for the rise of oxygen on Earth
}

\author{
Paul G. Falkowski
}

Published online: 29 December 2010

(C) Springer Science+Business Media B.V. 2010

\section{Introduction}

Oxygen is the third most abundant element in our solar system. Atomic oxygen is formed along the so-called 'main line' sequence from the high-temperature fusion of four ${ }^{4} \mathrm{He}$ atoms in hot stars. Upon the explosion of the star at the end of its natural lifetime, the oxygen and other elements are redistributed and ultimately reaccreted to form new planets around a new sun. All oxygen on Earth was obtained during this accretion process approximately 4.6 billion years ago (Clayton 1993). The concentration of oxygen is approximately equal to or slightly higher than that of carbon in the solar atmospheres in this region of our galaxy.

Molecular orbital calculations reveal that the atom has six valence electrons, a valence of two and naturally forms a diradical molecule with one $\sigma$ and one $\pi$ bond and two unpaired electrons in degenerate lower (anti-bonding) orbitals; hence the ground state of molecular $\mathrm{O}_{2}$ is a triplet. This unusual electron configuration prevents $\mathrm{O}_{2}$ from reacting readily with atoms or molecules in a singlet configuration without forming radicals (Valentine et al. 1995); however, reactions catalyzed by metals or photochemical processes often lead to oxides of group I, II, III, IV, V and even VI elements spanning $\mathrm{H}_{2} \mathrm{O}, \mathrm{MgO}$ and $\mathrm{CaO}, \mathrm{AlO}$, $\mathrm{CO}_{2}, \mathrm{SiO}_{2}, \mathrm{NO}_{x}, \mathrm{PO}_{4}$ and $\mathrm{SO}_{x}$. Oxygen also reacts with many trace elements, especially $\mathrm{Mn}$ and $\mathrm{Fe}$, which in aqueous phase forms insoluble oxyhydroxides at neutral

P. G. Falkowski ( $\square)$

Environmental Biophysics and Molecular Ecology Program, Department of Earth and Planetary Sciences and Institute of Marine and Coastal Sciences, Rutgers University,

New Brunswick, NJ 08901, USA

e-mail: falko@marine.rutgers.edu
$\mathrm{pH}$. The reactivity of oxygen is driven by electron transfer (redox) reactions, leading to highly stable products, such as $\mathrm{H}_{2} \mathrm{O}, \mathrm{CO}_{2}, \mathrm{HNO}_{3}, \mathrm{H}_{2} \mathrm{SO}_{4}$ and $\mathrm{H}_{3} \mathrm{PO}_{4}$. The abiotic reactions of oxygen often involve unstable reactive intermediates such as $\mathrm{H}_{2} \mathrm{O}_{2}, \mathrm{NO}, \mathrm{NO}_{2}, \mathrm{CO}$ and $\mathrm{SO}_{2}$. The reactions of oxygen with the other abundant light elements are almost always exergonic, meaning that, in contrast to $\mathrm{N}_{2}$, without a continuous source, free molecular oxygen would be depleted from Earth's atmosphere within a few million years (Falkowski and Godfrey 2008).

Earth is a unique planet in our solar system. Not only is it the only planet with both liquid water on its surface and sufficient radiogenic heat in its core to sustain plate tectonic processes, but its gas composition is far from thermodynamic equilibrium. Metaphorically the planet is similar to a gigantic biological cell. The analogue of a cell membrane is a thin film of crustal rock that separates the oxidized atmosphere on the outside from a reduced lithosphere on the inside. The energy sustaining this non-equilibrium condition is the photosynthetic transduction of solar energy to chemical bond energy.

Over the past $\sim 2.4$ billion years, oxygenic photosynthesis used liquid water as the dominant source of reductant, and carbon dioxide (or its hydrated equivalents) as the primary oxidant. The result over geological time has been the stable formation of molecular oxygen on the planetary surface. Indeed, at $\sim 4 \times 10^{18} \mathrm{~mol}, \mathrm{O}_{2}$ is the second most abundant gas in Earth's atmosphere. The origin, evolution, and mechanism of the water splitting reaction remain among the major unresolved questions in biology. However, although the photobiological splitting of water allowed for oxygenation of the atmosphere, geological processes were also critical; unless the reducing equivalents produced by photosynthetic organisms are buried in the lithosphere, there could not have been a net oxidation 
of the atmosphere, and without oxygen, complex animal life could not have arisen. In this special issue of Photosynthesis Research, we explore hypotheses related to the evolution of oxygenic photosynthesis, the geochemical evidence for the oxidation of Earth's atmosphere, and the consequences of the altered redox state to the Earth system, including the evolution of animal life.

\section{Biological contingencies}

All oxygenic photosynthetic organisms are derived from a single common ancestor, the origin of which remains obscure (Falkowski and Knoll 2007). The contemporary manifestation of this metabolic pathway in prokaryotes is restricted to a single taxa, cyanobacteria. All cyanobacteria contain two photochemical reaction centers, one which oxidizes water the second reduces ferredoxin. Despite large differences in the prosthetic groups and primary amino acid sequences between the two reaction centers, their molecular architecture is remarkably similar. While the two reaction centers appear to have originated from two extant clades of photosynthetic bacteria, molecular phylogeny and structural information suggest the two reaction centers themselves originated from a common ancestor, and diverged long before the origin of oxygenic photosynthesis (Sadekar et al. 2006). How and when the genes were transferred and mutated to yield an oxygenic photochemical apparatus is not clear. It is clear, however, that the manganese/calcium oxide cluster on the luminal side of photosystem II, and the four light driven electron transfer reactions leading to the production of each $\mathrm{O}_{2}$ molecule is unique in biology. The structure and evolution of PSII, is discussed by Hiller and his group (Williamson et al. 2010), and the timing of the appearance of cyanobacteria in the fossil record is discussed by Schopf (2010). The latter examines the data for both morphological fossils (or "cellular" fossils) as well as molecular fossils and isotopic measurements.

The oldest known rocks from which one potentially could infer early photosynthetic processes are from the Isua formation in southwest Greenland. Because of glacial scouring in the recent geological past, outcrops of these metamorphic rocks of clear sedimentary source are easily accessed, but because of post depositional heating they contain no morphological fossils. However, carbon, in the form of graphite from these rocks formed $\sim 3.8 \mathrm{Ga}$ (billion years ago) is isotopically depleted in ${ }^{13} \mathrm{C}$, strongly suggesting that the carbon was biologically derived from a photosynthetic pathway. Further, geochemical evidence of molecular biomarkers and morphological fossils suggest that cyanobacteria could have evolved as early as $3.2 \mathrm{Ga}$ or as late as $2.45 \mathrm{Ga}$, however, it seems that by about $2.4 \mathrm{Ga}$ sufficient oxygen accumulated in Earth's atmosphere to lead to the formation of stratospheric ozone.

The inference of ozone and the timing of this so-called "great oxidation event" (GOE) at 2.4 Ga comes primarily from analyses of sulfur isotopes in the rock record. The analyses and the interpretation, described by Farquhar et al. (2010) is based on the mass independent isotopic fractionation of sulfur. Basically, there are four stable sulfur isotopes, ${ }^{32} \mathrm{~S},{ }^{33} \mathrm{~S},{ }^{34} \mathrm{~S}$ and ${ }^{36} \mathrm{~S}$. Virtually all reactions that involve formation or the breaking of chemical bonds among these isotopes is mass-dependent, that is the isotope with the smaller mass is reactive (has a higher zero point kinetic energy) and the resulting products are predicted from first principles to be enriched in the lighter isotope. However, up until $\sim 2.4 \mathrm{Ga}$, the isotopic fractionations in the geologic record are mass independent. $\mathrm{SO}_{2}$ has a UV absorption cross section, peaking at $\sim 200 \mathrm{~nm}$. Breaking of bonds by high energy photons does not lead to mass dependent isotopic fractionation. Hence, one interpretation of the mass independent fractionation is that short wave UV radiation reached the Earth's surface prior to $\sim 2.4 \mathrm{Ga}$, but subsequently that radiation was quenched. Stratospheric ozone absorbs short wave UV radiation on the contemporary Earth, and the source of ozone is $\mathrm{O}_{2}$. Hence, the loss of the mass independent isotopic fractionation of sulfur at $2.4 \mathrm{Ga}$ suggests a change in the oxidation state of Earth's atmosphere. The mass independent fractionation signal for $\mathrm{S}$ never returned, and hence, it is concluded that the transition from an anaerobic world to an oxidized world occurred once, and only once, in Earth's history. It should be noted that the concentration of oxygen that arose during the GOE is extremely poorly constrained. Formation of stratospheric ozone is not limited by $\mathrm{O}_{2}$ above ca. $0.1 \%$ of the present atmospheric level. Geochemists use other proxies, including $\mathrm{N}$ isotopes (Godfrey and Falkowski 2009), transition metal composition and isotopic values (Kaufman et al. 2007) and even mineral composition (Hazen et al. 2008) to further attempt to constrain the concentration of oxygen during the GOE and to understand what controlled the net accumulation of the gas over the ensuing 2.3 billion years.

\section{Geological contingencies}

High concentrations of free molecular oxygen in a planetary atmosphere cannot come about simply by high energy photolysis of water; that reaction is self quenching as UV becomes increasingly blocked. Further, as in all redox reactions, a reductant (the equivalent of hydrogen) is formed. To bring about a change in the oxidation state of the atmosphere, the redox reactions cannot be at equilibrium, but rather the reductant has to be removed and stored 
for long periods of geological time. Hence, the evolution of oxygenic photosynthesis was a necessary, but not sufficient condition for the oxidation of the planetary surface.

In a simple geochemical sense, net production of oxygen on Earth implies the burial and sequestration of reductant. The reduced equivalents are primarily in the form of organic matter. Organic matter in the ocean is depleted in ${ }^{13} \mathrm{C}$ by $\sim 20 \%$ relative to the (arbitrarily chosen) standard, carbon from fossil (extinct) marine Belemnite carbonates in the Pee Dee formation in South Carolina (the PDB standard). By definition, the isotopic value of the standard relative to itself is $0 \%$. Mantel carbon, emitted from volcanoes, has an isotopic value of ca. $-5 \%$. Hence, to obtain such a mantel carbon isotopic value requires mixing 4 mass equivalents of carbonate with one mass equivalent of organic carbon. This basic notion provides the basis for estimating the oxidation state of the planetary surface (from a practical purpose, the atmosphere, as a very small fraction of the free oxygen is dissolved in the ocean or is found in crustal rocks). The notional concept is that as more organic carbon is buried oxygen concentrations in the atmosphere increase. On geological time scales, the burial of organic carbon removes the lighter isotope, ${ }^{12} \mathrm{C}$, in the inorganic phase, from the ocean/atmosphere system, leaving behind inorganic carbon that is increasingly enriched in ${ }^{13} \mathrm{C}$. Hence, on geological time scales, increased net oxidation of the Earth's surface can quantitatively be related to increased ${ }^{13} \mathrm{C}$ content of inorganic carbon buried in the rock record as carbonates. The geochemical record of carbon isotopes over geological time, while clearly not perfect, is extensive and clearly reveals the pattern of burial of reducing equivalents over the past 3.5 billion years. The results strongly suggest that organic carbon was extensively buried for 200 million years around the time of the GOE, and subsequently around $700 \mathrm{Ma}$ (million years ago), and $350 \mathrm{Ma}$.

Burial of organic matter on geological time scales is not trivial. Although until approximately $400 \mathrm{Ma}$, all primary production on Earth was confined to aquatic ecosystems (by far the oceans), and the residence time of marine sediments is relatively short-on order of ca. 200-300 million years. The sediments are largely subducted into the upper mantel where they are heated and the resulting gases emitted via volcanism back to the atmosphere. Indeed on geological time scales this is the source of $\mathrm{CO}_{2}$ in Earth's atmosphere. This so-called Wilson cycle [named after the late Canadian geophysicist, Tuozo Wilson (1966)] constrains oxidation of the atmosphere to small levels of oxygen, on order of ca. 1\% PAL. To escape this constraint, organic carbon must be removed from the cycle. One mechanism is the uplift of marine sediments onto continental cratons, where it can be stored for billions of years. Indeed, subduction of marine crust along active continental margins leads to the formation of stable sedimentary rocks (as shales and mudstones) uplifted onto land and hence removed from the Wilson cycle. This process is driven by plate tectonics. Earth is the only planet in our solar system with active plate tectonics. Hence, the combination of the evolution of oxygenic photosynthesis and plate tectonics has permitted this planet to escape an anaerobic condition and evolve to become extremely highly oxidized (Falkowski and Godfrey 2008).

Some additional organic material may be further subducted deeper into the mantle where, under high temperature and pressure it can be converted into highly stable forms including diamond. The deep subsurface carbon cycle is poorly understood, but viable microbes are found several kilometers in the interior, using organic carbon sources of which a fraction must have been produced photosynthetically hundreds of millions of years ago. Less than $0.1 \%$ of the organic matter formed at the Earth's surface is buried in the lithosphere. Given an atmospheric concentration of oxygen of $4 \times 10^{18} \mathrm{~mol}$, and assuming a steady-state model, it is estimated that the turnover of $\mathrm{O}_{2}$ is about $4 \times 10^{6}$ years.

\section{Biogeochemical consequences}

The geochemical consequences of the oxidation of Earth's atmosphere and oceans were profound. The oxidation altered many biogeochemical cycles, not the least being that of nitrogen. With the availability of free molecular oxygen, ammonium could be oxidized to nitrite and nitrate by chemoautrophic bacteria, and the oxidized forms of nitrogen, could in turn, be reduced to $\mathrm{N}_{2} \mathrm{O}$ and $\mathrm{N}_{2}$ by facultative anaerobes. Thus the $\mathrm{N}$ cycle would accelerate by a factor of approximately $10^{4}$ leading to an explosive potential to enhanced primary production in the oceans. Indeed, over the ensuing several hundred million years following the GOE, cyanobacteria were serially transferred to several clades of eukaryotic cells, one of which became the founder species for all terrestrial plants. The diversity of eukaryotic algae is enormous, and experimental endosymbiotic events occur continuously; this topic is discussed by both Green (2010) and Johnson (2010). The experimentation in endosymbiotic associations led to several types of antenna chlorophyll protein complexes serving highly conserved reaction center cores. Indeed, the D1 protein, integral to the reaction center of PSII, only has $14 \%$ variability at the amino acid level from cyanobacteria to oak trees. The reaction center proteins are extreme examples of "frozen metabolic accidents"-structures adapted from anaerobic photosynthetic organisms and recycled in oxygenic photosynthesis. This issue is addressed in this volume by Allen and Williams (2010). 
The evolution of eukaryotic algae had a further feedback on the evolution of the oxidation state of Earth. Being larger cells, they tend to sink much faster than cyanobacteria, and hence accelerate the export and burial efficiency of organic matter in marine sediments. This acceleration almost certainly helped bring about a rise in oxygen in the late Paleoproterozoic and early Cambrian ( $\sim 600$ million years ago), allowing the rise of multicellular animals. Indeed, the Cambrian "explosion" was probably enabled by the evolution of eukaryotic algae. The rise of oxygen and its effects on animal size and complexity is discussed by Payne et al. (2010).

\section{Concluding remarks}

A naive chemist examining the atmosphere on Earth may be completely surprised that the two most abundant gases are $\mathrm{N}_{2}$ and $\mathrm{O}_{2} . \mathrm{N}_{2}$ behaves as a noble gas and it is virtually non-reactive. Geochemists assume that the amount of $\mathrm{N}_{2}$ in the atmosphere has remained constant since the planet was formed. Indeed, the turnover time for $\mathrm{N}_{2}$ in the atmosphere is estimated to be $\sim$ a billion years (Berner 2006). In contrast, $\mathrm{O}_{2}$, exists far from thermodynamic equilibrium and has a turnover time on order of a few million years (Keeling et al. 1993). Indeed, high concentrations of gaseous diatomic oxygen are unique to this planet in our solar system and this feature of our planetary atmosphere has not yet been found on any other planet within approximately 20 parsecs of us. The presence of high concentrations of the gas in a planetary atmosphere is presently understood to be a virtually irrefutable indication of life on other terrestrial planets. Why is the gas so abundant on Earth yet so scarce on other planets in our solar system and apparently beyond? Those questions remain fundamental to our understanding of the evolution of oxygenic photosynthesis on Earth.

Acknowledgments My research on the oxygen cycle is supported by NASA, NSF, and the Agouron Institute.

\section{References}

Allen JP, Williams JC (2010) The evolutionary pathway from anoxygenic to oxygenic photosynthesis examined by comparison of the properties of photosystem II and bacterial reaction centers. Photosynth Res. doi:10.1007/s11120-010-9552-x

Berner R (2006) Geological nitrogen cycle and atmospheric N2 over Phanerozoic time. Geology 34:413-415

Clayton R (1993) Oxygen isotopes in meteorites. Annu Rev Earth Planet Sci 21:115-149

Falkowski PG, Godfrey L (2008) Electrons, life, and the evolution of earth's oxygen cycle. Philos Trans R Soc 363:2705-2716

Falkowski PG, Knoll A (eds) (2007) Evolution of primary producers in the sea. Academic Press, New York, pp 441

Farquhar J, Zerkle AL et al (2010) Geological constraints on the origin of oxygenic photosynthesis. Photosynth Res. doi:10.1007/ s11120-010-9594-0

Godfrey L, Falkowski PG (2009) The cycling and redox state of nitrogen in the Archean ocean. Nat Geosci 2:725-729

Green B (2010) After the primary endosymbiosis: an update on the chromalveolate hypothesis and the origins of algae with Chl c. Photosynth Res. doi:10.1007/s11120-010-9584-2

Hazen RM et al (2008) Mineral evolution. Am Mineral 93:1693-1720

Johnson MD (2010) The acquisition of phototrophy: adaptive strategies of hosting endosymbionts and organelles. Photosynth Res. doi:10.1007/s11120-010-9546-8

Kaufman AJ, Johnston DT, Farquhar J, Masterson AL, Lyons TW, Bates S, Anbar AD, Arnold GL, Garvin J (2007) Late Archean biospheric oxygenation and atmospheric evolution. Science 317: 1900-1903

Keeling R et al (1993) What atmospheric oxygen measurements can tell us about the global carbon cycle. Glob Biogeochem Cycles 7: $37-67$

Payne JL et al (2010) The evolutionary consequences of oxygenic photosynthesis: a body size perspective. Photosynth Res. doi: 10.1007/s11120-010-9593-1

Sadekar S et al (2006) Conservation of distantly related membrane proteins: photosynthetic reaction centers share a common structural core. Mol Biol Evol 23:2001-2007

Schopf JW (2010) The paleobiological record of photosynthesis. Photosynth Res. doi:10.1007/s11120-010-9577-1

Valentine J et al (1995) Active oxygen in biochemistry. Chapman and Hall, London

Williamson A et al (2010) The evolution of Photosystem II: insights into the past and future. Photosynth Res. doi:10.1007/s11120010-9559-3

Wilson JT (1966) Did the Atlantic close and then re-open? Nature 211:676-681 